\title{
Editorial
}

\section{New Insights for Improving Hand Hygiene Practices}

\author{
John M. Boyce, MD
}

Publication of the Guideline for Hand Hygiene in Health-Care Settings, developed by the Healthcare Infection Control Practices Advisory Committee (HICPAC), the Society for Healthcare Epidemiology of America (SHEA), the Association for Professionals in Infection Control (APIC), and the Infectious Diseases Society of America (IDSA), ${ }^{1}$ has stimulated interest in improving hand hygiene practices in healthcare facilities. Endorsement of the Guideline by the Joint Commission on Accreditation of Healthcare Organizations should serve as additional impetus for institutions to develop and implement multidisciplinary programs to increase adherence of healthcare workers ( $\mathrm{HCWs)}$ to recommended hand hygiene measures. ${ }^{2}$ Although the Guideline provides a comprehensive review of published evidence on the subject, there is still much to be learned about hand hygiene practices and how to improve them. Several articles ${ }^{3-7}$ appearing in this issue of Infection Control and Hospital Epidemiology provide new insights that should be incorporated into hand hygiene improvement programs.

For approximately 30 years, it has been assumed that the importance of handwashing or hand antisepsis (using antimicrobial soap and water or an alcohol handrub) depended to some extent on the type of patient care activity being performed. In the 1970s, Fulkerson described 15 types of activities that were assumed to cause increasing degrees of hand contamination. ${ }^{8}$ Activities ranked 1 to 4 or 1 to 6 were considered "clean procedures" and those with higher rankings were classified as "dirty procedures." However, the Fulkerson scale has not been validated by culturing HCWs' hands following each of the 15 categories of contact. One earlier study that evaluated several types of patient care activities established that the rate and degree of hand contamination does vary for different types of contact. ${ }^{9}$ In this issue of Infection Control and Hospital Epidemiology, several articles provide additional data on contamination of HCW' hands and the frequency with which hands are cleaned after various patient care activities.
In a study conducted in a neonatal intensive care unit, Pessoa-Silva et al. $^{3}$ established that hand contamination was greatest during diaper changes and respiratory care, followed by direct skin contact and contact with other types of body secretions. These findings help validate the ranking of procedures described in the Fulkerson scale. However, they demonstrated that some clean procedures (eg, contact with inanimate objects in the vicinity of a patient) may cause hand contamination. The latter finding confirms earlier studies conducted in different patient care settings. ${ }^{9-12}$ The authors found that colony counts on the hands of HCWs caring for neonates increased an average of 24.5 colony-forming units per minute when gloves were not worn. ${ }^{3}$ Hands became contaminated with up to 100 colony-forming units after only 2 minutes of respiratory care, changing diapers, or having direct skin contact. Wearing gloves reduced, but did not eliminate, hand contamination, a finding reported by others. ${ }^{9,10,13,14}$ The authors also emphasized the need to clean hands between sequences of care performed on the same patient, as recommended in the Guideline. ${ }^{1}$

The study by Wendt et al. ${ }^{5}$ in this issue of Infection Control and Hospital Epidemiology determined the frequency with which HCWs cleaned their hands with an alcohol handrub following the 15 types of patient activities described by the Fulkerson scale. Of interest, the authors found that the greater the predicted risk of hand contamination, the more likely $\mathrm{HCW}$ s were to clean their hands. This observation provides new evidence that the Fulkerson scale correlates fairly well with HCWs' attitudes about the need to clean hands after various activities, as suggested in earlier studies. ${ }^{15}$ However, several findings in the study by Wendt et al. were of concern. Some nurses spent time unnecessarily cleaning their hands after activities with little risk of contamination. Also, intensive care unit personnel performed hand antisepsis less than $40 \%$ of the time when having direct contact with patient secretions or contact with objects contaminated with patient secretions. 
Raboud et al. $^{4}$ reported that nurses visited patient rooms more than 50 times during a work shift, and $76 \%$ of such visits involved activities for which hand hygiene is recommended. These findings emphasize the need to provide HCWs with hand hygiene products that are well tolerated despite frequent application. These authors, similar to Wendt et al.,5 observed that the frequency with which HCWs performed hand hygiene varied, depending on the perceived risk of hand contamination. Hand antisepsis using an alcohol handrub was performed after contact with body fluids $81 \%$ of the time, somewhat less often after skin contact (61\%), and even less often after contact with environmental surfaces $(38 \%)$. It is somewhat encouraging that HCWs performed hand antisepsis following dirty procedures more frequently in the studies by Raboud et al. ${ }^{4}$ and Wendt et al. ${ }^{5}$ than in an earlier study in which nurses washed their hands only $12 \%$ of the time following dirty procedures. ${ }^{8}$ However, it appears that a substantial proportion of our colleagues are still not aware that hand contamination can occur when having direct contact with a patient's intact skin, and that hand antisepsis is indicated following such activities. Interestingly, although alcohol handrubs had been available for a matter of years in both study institutions, the overall rate of adherence to recommended hand hygiene procedures was $65 \%$ in the study by Wendt et al. versus $46 \%$ in the study by Raboud et al. From the information provided, it is not clear whether the disparity is due to differences in study design, criteria for adherence, effectiveness of educational efforts, level of institutional support, or satisfaction of HCWs with the hand rinse product available.

The article by Widmer and Dangel ${ }^{6}$ in this issue of Infection Control and Hospital Epidemiology addresses another important issue-the quality of the hand hygiene technique practiced by HCWs. It is well established that HCWs often fail to perform handwashing using recommended techniques. Numerous studies have documented that $\mathrm{HCW}$ s frequently do not wash their hands with soap and water for the recommended amount of time. ${ }^{1}$ Other aspects of handwashing technique, such as cleaning all surfaces of the hands and fingers, are often performed poorly. ${ }^{8,16,17}$

Despite the increasing use of alcohol handrubs in healthcare facilities, little is known about how well HCWs clean their hands when using such products. In one clinical study, unexpectedly poor efficacy of alcohol handrubs in reducing bacterial counts on hands was attributed to poor technique, although no formal observations were recorded. ${ }^{18}$ The study by Widmer and Dangel ${ }^{6}$ is the first to use objective, quantitative methods to evaluate the technique used by HCWs (infection control personnel) to apply an alcohol hand rinse, and to correlate the observations with bacterial cultures of hands before and after the procedure. On average, bacterial counts decreased by $99 \%\left(2 \log _{10}\right)$. However, for $25 \%$ of the participants, the log reduction achieved was less than $1.1 \log _{10}$, approximately the same as that achieved by washing hands with plain (non-antimicrobial) soap and water. Participants who worked in facilities where an alcohol handrub was already in use did no better than those working in hospitals where handwashing was still the norm. The authors' findings highlight the need to provide HCWs with specific education on how best to apply alcohol handrubs.

Training techniques that should be considered include real-time visual assessments of how well the individual applied the handrub, or agar plate hand-print cultures performed before and after applying the product.

Finally, the article by Gupta et al. ${ }^{7}$ in this issue of Infection Control and Hospital Epidemiology provides additional epidemiologic evidence that wearing artificial fingernails may be associated with transmission of healthcare-associated pathogens. This study, combined with earlier in vivo studies and epidemiologic investigations, supports the recent recommendation that $\mathrm{HCWs}$ who have direct contact with high-risk patients should not wear artificial fingernails. ${ }^{1}$

\section{REFERENCES}

1. Boyce JM, Pittet D. Guideline for hand hygiene in health-care settings: recommendations of the Healthcare Infection Control Practices Advisory Committee and the HICPAC/SHEA/APIC/IDSA Hand Hygiene Task Force. $M M W R$ 2002;51(RR16):1-44

2. Joint Commission on Accreditation of. Healthcare Organizations. Sentinel Event Alert. Oakbrook Terrace, IL: Joint Commission on Accreditation of Healthcare Organizations; 2003. Available at www.jcaho.org/about+us/news+letters/sentinel+event+alert/sea_28.htm. Accessed February 20, 2004.

3. Pessoa-Silva $\mathrm{CL}$, Dharan S, Hugonnet $\mathrm{S}$, et al. Dynamics of bacterial hand contamination during routine neonatal care. Infect Control Hosp Epidemiol 2004;25:192-197.

4. Raboud J, Saskin R, Wong K, et al. Patterns of handwashing behavior and visits to patients on a general medical ward of healthcare workers. Infect Control Hosp Epidemiol 2004;25:198-202.

5. Wendt $\mathrm{C}$, Knautz D, von Baum $\mathrm{H}$. Differences in hand hygiene behavior related to the contamination risk of healthcare activities in different groups of healthcare workers. Infect Control Hosp Epidemiol 2004;25:203-206

6. Widmer AF, Dangel M. Alcohol-based handrub: evaluation of technique and microbiological efficacy with international infection control professionals. Infect Control Hosp Epidemiol 2004;25:207-209.

7. Gupta A, Della-Latta P, Todd B, et al. Outbreak of extended-spectrum beta-lactamase-producing Klebsiella pneumoniae in a neonatal intensive care unit linked to artificial nails. Infect Control Hosp Epidemiol $2004 ; 25: 210-215$.

8. Fox MK, Langner SB, Wells RW. How good are hand washing practices? Am J Nurs 1974;74:1676-1678.

9. Pittet D, Dharan S, Touveneau S, Sauvan V, Perneger TV. Bacterial contamination of the hands of hospital staff during routine patient care. Arch Intern Med 1999;159:821-826.

10. McFarland LV, Mulligan ME, Kwok RYY, Stamm WE. Nosocomial acquisition of Clostridium difficile infection. $N$ Engl J Med 1989;320:204210.

11. Boyce JM, Potter-Bynoe G, Chenevert C, King T. Environmental contamination due to methicillin-resistant Staphylococcus aureus: possible infection control implications. Infect Control Hosp Epidemiol 1997;18:622627.

12. Samore MH, Venkataraman I, Degirolami PC, Levin E, Karchmer AW. Clinical and molecular epidemiology of sporadic and clustered cases of nosocomial Clostridium difficile diarrhea. Am J Med 1996;100:32-40.

13. Tenorio AR, Badri SM, Sahgal NB, et al. Effectiveness of gloves in preventing personnel hand carriage of vancomycin-resistant enterococcus (VRE) after patient care. Clin Infect Dis 2001;32:826-829.

14. Lucet JC, Rigaud MP, Mentre F, et al. Hand contamination before and after different hand hygiene techniques: a randomized clinical trial. $J$ Hosp Infect 2002;50:276-280.

15. Iarson E, Johnson RW, Lusk E. Evaluating handwashing technique. $J$ Adv Nurs 1985;10:547-552.

16. Taylor LJ. An evaluation of handwashing techniques-1. Nursing Times 1978:74:54-55.

17. Gould D, Ream E. Assessing nurses' hand decontamination performance. Nursing Times 1993;89:47-50.

18. Ojajarvi J. Handwashing in Finland. J Hosp Infect 1991;18:35-40. 